\title{
TRATAMENTO ESTÉTICO ANTERIOR: ASSOCIAÇÃO DE CLAREAMENTO DENTAL E SUBSTITUIÇÃO DE RESTAURAÇÕES INSATISFATÓRIAS
}

\author{
ANTERIOR AESTHETIC TREATMENT: ASSOCIATION OF TEETH \\ WHITENING AND REPLACEMENT OF UNSATISFACTORY RESTORATIONS
}

Alvaro Augusto Junqueira Júnior ${ }^{1}$, Camila Imperador Rodrigues Alves ${ }^{2}$, Marcelo Rodrigues Alves ${ }^{1}$, Paulo André Yamin', Pablo Leal Teixeira Santos ${ }^{3}$, Aline Akemi Mori ${ }^{*}$.

1 Universidade de São Paulo, Ribeirão Preto, SP, Brasil.

2Universidade Estadual de Campinas, Campinas, SP, Brasil.

3UNINGÁ - Centro Universitário Ingá, Maringá, PR, Brasil.

*alinemori.odonto@gmail.com

Recebido em: 28/11/2019; Aceito em: 02/12/2020.

\section{RESUMO}

Um dos maiores desafios da odontologia minimamente invasiva é o reestabelecimento restaurador de tecidos dentais perdidos ao mesmo tempo em que se preserva ao máximo os substratos sadios, sem realizar desgastes adicionais em esmalte e dentina hígidos. Dentre os procedimentos odontológicos estéticos, o clareamento dental é um dos mais requisitados pelos pacientes insatisfeitos com a aparência do seu sorriso. Isto ocorre devido a sua eficácia, baixa invasividade e simplicidade da técnica. Após a realização do procedimento, se faz necessário a substituição das restaurações pré-existentes em razão do aspecto não harmônico resultante. Este trabalho tem como objetivo relatar um caso clínico de tratamento estético anterior em que os anseios estéticos da paciente foram resolvidos sob uma ótica conservadora, minimante invasiva com clareamento dental (técnica associada) e restaurações tipo Classe III e IV em resina composta. Para que o tratamento tenha sucesso, todas as etapas clínicas são de suma importância e, neste caso, as expectativas do paciente foram alcançadas por meio de uma abordagem conservadora e com alta previsibilidade.

Palavras-chave: Clareamento dental. Falha de restauração dentária. Restauração dentária.

\section{ABSTRACT}

One of the greatest challenges of minimally invasive dentistry is the restoration of lost dental tissues while preserving healthy substrates to the maximum extent, without additional wear on healthy enamel and dentin. Among aesthetic dental procedures, teeth whitening is one of the most requested by patients who are unsatisfied with their smiles' appearance. This is due to the effectiveness, low invasiveness, and simplicity of the whitening technique. After performing the procedure, it is necessary to replace any pre-existing restorations given the resulting non-harmonic aspect of the teeth. This paper aims to report on a clinical 
case of anterior aesthetic treatment in which the patient's aesthetic desires were met following a conservative, minimally invasive approach with teeth whitening (associated technique), and Class III and IV restorations in composite resin. In order for the treatment to be successful, all clinical steps are of paramount importance and, in this case, the patient's expectations were achieved through a conservative approach and with high predictability.

Keywords: Dental restoration. Dental restoration failure. Teeth whitening.

\section{INTRODUÇÃO}

A alta exigência estética dos pacientes nos últimos anos tem impulsionado o desenvolvimento de diferentes técnicas e materiais restauradores, o que possibilitou a expansão das possibilidades de tratamentos oferecidos pela Odontologia (BARATIERI, 2001; JOINER; LUO, 2017). O clareamento dental é um dos procedimentos mais procurados, pois está associado à estética e à saúde bucal. Por ser um procedimento conservador e que não impossibilita outras formas de tratamento, o clareamento tornou-se a primeira escolha para tratamento de dentes com alteração de cor (MAIA et al., 2008; QUAGLIATTO, 2020).

Dentre as diversas técnicas de disponíveis para este procedimento, as mais utilizadas são o clareamento dental de consultório ("office"), com altas concentrações de peróxidos, e o clareamento caseiro, realizado pelo paciente e sob supervisão do Cirurgião-Dentista com peróxidos de baixas concentrações (JOINER, 2006; JOINER; LUO, 2017). O peróxido de hidrogênio age quando em contato com os dentes e a saliva, atuando como forte oxidante. Moléculas de baixo peso molecular penetram no esmalte e na dentina, atingindo cromóforos compostos por cadeias moleculares longas e de ligações insaturadas, com alto índice de absorção de luz. A ação oxidante do agente clareador é capaz de quebrar tais moléculas em cadeias menores, produzindo compostos menos comogênicos e obtendo, assim, um efeito clareador (DAHL; PALLESEN, 2003).

Os avanços alcançados em relação à adesão aos tecidos dentais (VAN MEERBEEK et al., 2010) e a evolução dos polímeros dentários possibilitou a realização de restaurações conservadoras em dentes anteriores de maneira rápida, segura e esteticamente agradável. Ainda hoje, a realização de novas restaurações ou a substituição de restaurações antigas é um dos procedimentos mais comuns e que consomem mais tempo clínico na rotina do CirurgiãoDentista (MJOR et al., 2002). A resina composta tornou-se um material restaurador largamente utilizado por devolver eficientemente a estética com diminuição da necessidade de remoção de tecido dental sadio (FERRACANE, 2011), passando a ser, assim, a primeira escolha de material para restaurações anteriores e posteriores (DEMARCO et al., 2013). Ao contrário das restaurações posteriores, que muitas vezes são substituídas devido à ocorrência de cáries secundárias, as restaurações anteriores são substituídas, na maioria das vezes, por razões estéticas (DEMARCO et al., 2015). Apesar do aumento do sucesso clínico destas restaurações ao longo dos anos, Cirurgiões-Dentistas parecem andar na contramão da filosofia de mínima intervenção: vivemos uma realidade profissional em que tratamentos demasiadamente invasivos estão sendo 
propostos para situações clínicas outrora bem resolvidas com restaurações conservadoras.

Este trabalho tem como objetivo relatar um caso clínico de tratamento estético anterior em que os anseios estéticos da paciente foram resolvidos sob uma ótica conservadora, com clareamento dental e restaurações tipo Classe III e IV em resina composta.

\section{RELATO DE CASO}

Paciente de 55 anos, gênero feminino, queixava-se da estética do sorriso, forma e a cor dos dentes e restaurações apresentavam-se inadequadas (Figura 1). A paciente foi instruída da necessidade de substituição das restaurações anteriores do tipo Classe III e IV, visto que estas se apresentavam manchadas, infiltradas e com estratificação inadequada. Foi proposta a realização de clareamento dental como primeiro procedimento estético, a fim de diminuir a saturação inicial (dentes com cor A4 na Escala Vita Classic, Figura 2).

Figura 1 - Aspecto intraoral inicial

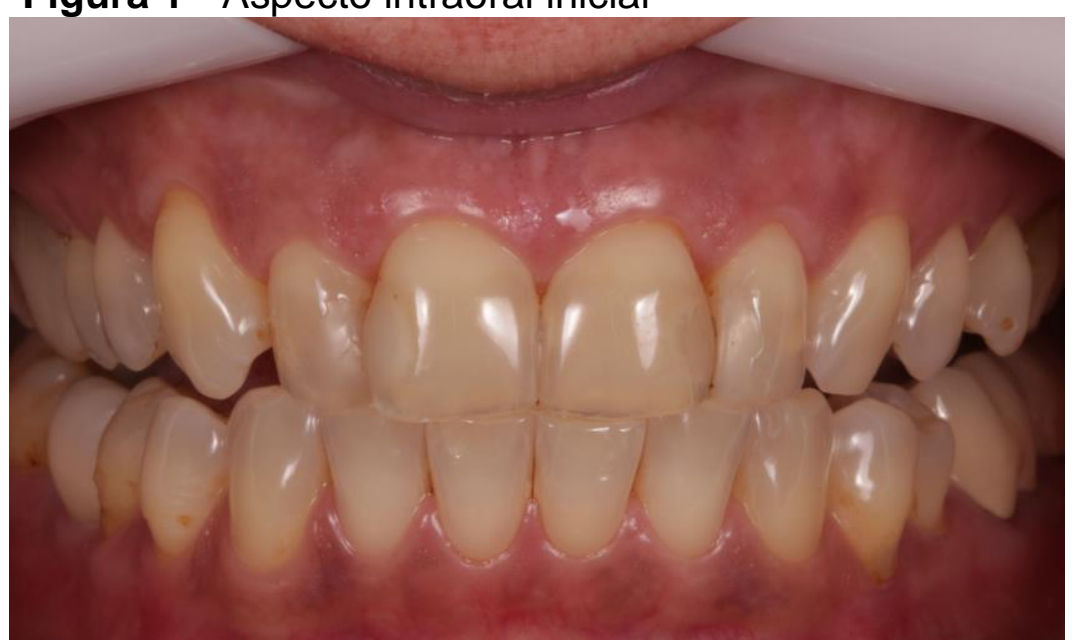

Fonte: os autores.

Figura 2 - Tomada de cor inicial, previamente ao tratamento clareador

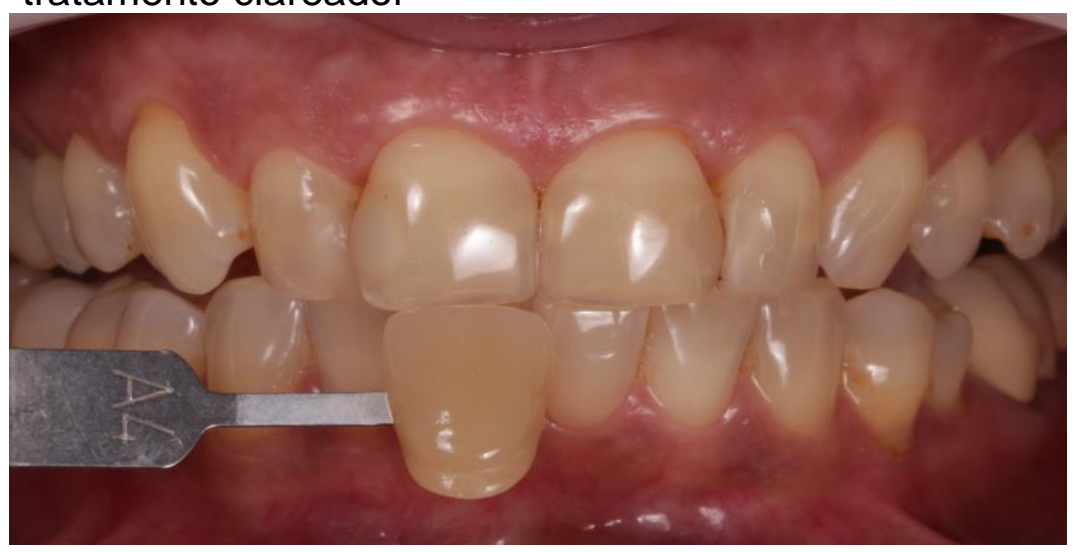

Fonte: os autores. 
Foi escolhida a técnica associada de clareamento dental, pois o tempo geralmente necessário para o clareamento dental caseiro é de 3 a 4 semanas, 0 que não seria vantajoso para os anseios pessoais da paciente. Na técnica associada, o clareamento de consultório com gel clareador em alta concentração é realizado por uma ou duas sessões, sendo associado ao clareamento caseiro com gel clareador de baixa concentração com o auxílio de moldeiras. O clareamento foi iniciado com uma sessão de consultório com peróxido de hidrogênio a 37,5\% (Pola Office Plus, SDI, Austrália), com 3 aplicações de 15 minutos cada, sem aplicação de fonte de luz.

A paciente continuou o clareamento caseiro com peróxido de carbamida a 16\% (Whiteness Perfect, FGM, Brasil), com aplicações de $2 \mathrm{~h}$ diárias. Ao término do período de clareamento caseiro, uma segunda e última sessão se clareamento de consultório foi realizada, seguindo o mesmo protocolo da primeira sessão. Quatorze dias após, a paciente retornou ao consultório para o tratamento restaurador anterior. A figura 3 mostra o resultado final do clareamento, ainda sem a substituição das restaurações, onde houve diminuição da saturação cromática e obteve-se a tonalidade A2 da Escala Vita Classic.

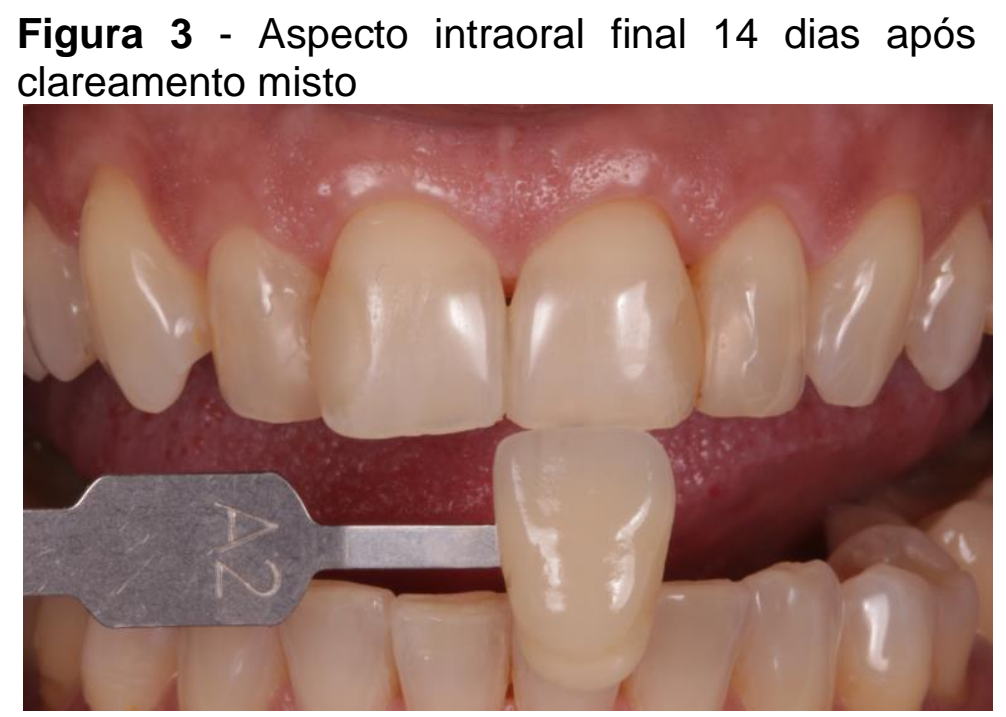

Fonte: os autores.

Com o fim da etapa de clareamento, as restaurações anteriores deficientes mostraram-se ainda mais insatisfatórias do ponto de vista estético. Todas as restaurações anteriores foram trocadas na mesma consulta. Como todas as cavidades envolviam ângulo de reflexão, foram confeccionados biseis nas margens das cavidades por vestibular, de modo a tornar a transição da restauração com o remanescente dental menos perceptível esteticamente. $O$ procedimento adesivo foi pela técnica do condicionamento ácido total: aplicação de ácido fosfórico 35\% (Ultraetch, Ultradent, EUA) por 30 segundos em esmalte e 15 segundos em dentina, lavagem copiosa com água e ar por 30 segundos, aplicação de sistema adesivo para dentina e esmalte (Optibond FL, Kerr, EUA) e fotopolimerização por 40 segundos.

As restaurações proximais foram estratificadas com resinas suprananométricas de esmalte e dentina (Palfique LX5, Tokuyama, Japão), sendo a camada de resina mais opaca (de dentina) estendida até pouco mais da 
metade da área do bisel. A área coberta por resina opaca no bisel deve variar de acordo com as características ópticas de cada sistema de resina composta.

A resina Palfique LX5 tem como característica baixa opacidade das resinas para dentina. Com isso, uma espessura maior deste tipo de resina se faz necessária para evitar um acinzentamento da restauração, consequência indesejável e recorrente quando a restauração final apresenta baixa opacidade. Também foram restauradas as bordas incisais dos incisivos centrais com resina nanohíbrida (Forma, Ultradent, EUA), que estavam desgastadas e apresentando curvatura invertida. Estes acréscimos tornam a curvatura do sorriso mais harmônica e restabelecem correta guia protrusiva. A troca das restaurações antigas e os acréscimos incisais permitiram um tratamento cosmético com o mínimo de desgaste dental possível. (Figura 4) O acabamento e polimento foi realizado com discos abrasivos (Sof-Lex Pop On, 3M-ESPE, EUA) e taças de borracha (Astropol, Ivoclar-Vivadent, Lichtenstein).

O aspecto final pode ser melhor visualizado pela foto extra oral de sorriso, que evidencia harmonia de forma e cor dos dentes, bem como uma relação esteticamente mais agradável das bordas incisais com o inferior. (Figura 5)

Figura 4 - Aspecto intraoral final, após a troca das restaurações antigas deficientes e restabelecimento das bordas incisais dos incisivos centrais

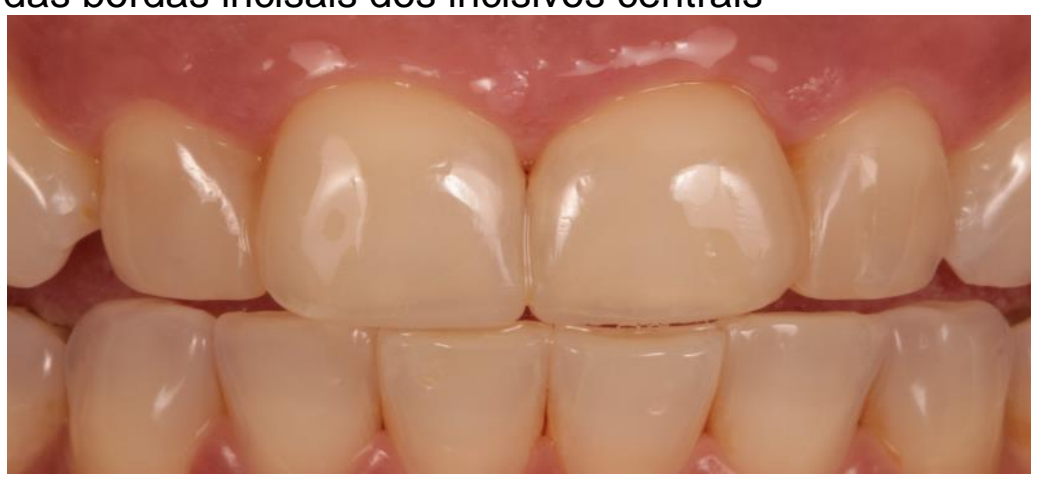

Fonte: os autores.

Figura 5 - Aspecto final do sorriso

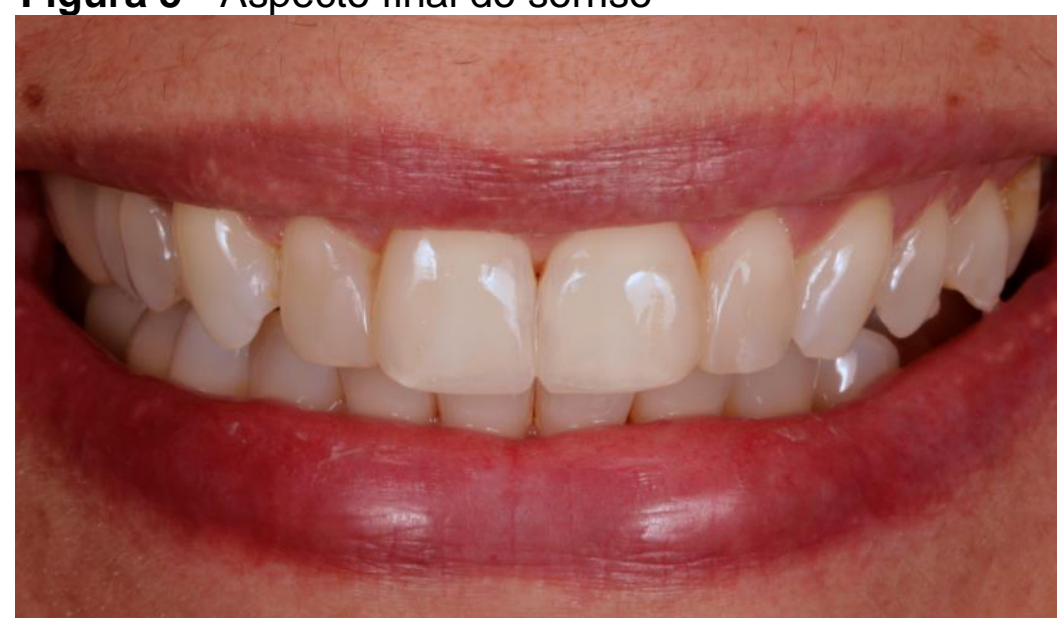

Fonte: os autores. 


\section{DISCUSSÃO}

O tratamento restaurador com resina composta permite a devolução de forma, contorno, cor e textura dos dentes, sendo amplamente utilizado na odontologia (DEMARCO et al., 2015). São considerados esteticamente satisfatórios quando são capazes de mimetizar a aparência do substrato dental a qual é submetido. Nas resinas compostas, isto é alcançado associando a técnica precisa à escolha e estabilidade da cor do material utilizado ( $\mathrm{CHOI}$ et al., 2005).

Uma das principais causas de falha em restaurações diretas em dentes anteriores é a aparência insatisfatória relacionada a instabilidade da cor tanto do material restaurador quanto do próprio substrato dental, pois ambos podem sofrer modificações ao longo do tempo (DEMARCO et al., 2015). Isto ocorre devido a absorção de pigmentos presentes no meio bucal, oriunda de alimentos como café, refrigerante do tipo cola, vinho tinto, chá e uísque (PATEL et al., 2004; ABU-BAKR et al., 2005; BAGUERI; BURROW; TYAS, 2005; ERTAS et al., 2006) por um fator intrínseco inerente ao material (UCHIDA et al., 1998; FONTES et al., 2009), condição do polimento final, tipo de resina utilizado e manchas extrínsecas (FONTES et al., 2009).

O tratamento clareador deve ser considerado sempre que tratamentos estéticos anteriores forem propostos ao paciente. A existência de restaurações prévias e a possibilidade de novos tratamentos restauradores influenciam no planejamento clareador, visto que restaurações antigas podem tornar-se mais evidentes após o clareamento, enquanto restaurações futuras devem ser confeccionadas baseando-se na cor obtida ao fim desta etapa (HIRATA, 2016).

Considerando a idade da paciente, o resultado obtido com o protocolo executado foi dentro do esperado. Isto porque efeito um clareador mais expressivo geralmente está associado a pacientes mais jovens (REZENDE et al., 2016). O emprego de peróxido de hidrogênio no consultório em 3 aplicações de 15 minutos teve como objetivo evitar queda considerável no $\mathrm{pH}$ do gel, o que poderia causar sensibilidade pós-operatória exacerbada (KOSE et al., 2015). Não foi utilizada fonte de luz, pois as evidências científicas atuais mostram que seu emprego não melhora o efeito clareador (MARAN et al., 2019). Não foi recomendado à paciente evitar o consumo de alimentos e bebidas com corantes, pois os trabalhos clínicos mais recentes não encontraram relação entre seu consumo e a qualidade final do tratamento clareador (REZENDE et al., 2013; MATIS et al., 2015; MORI et al., 2016).

O intervalo de 2 semanas entre o fim do tratamento clareador e os procedimentos restauradores é importante para garantirmos que o oxigênio residual do clareamento terá sido completamente eliminado. A realização de procedimentos adesivos antes deste período é um risco, pois tal oxigênio residual pode interferir na adequada polimerização do sistema adesivo. (MCGUCKIN; THURMOND; OSOVITZ, 1992; TITLEY et al., 1993).

Os princípios de retenção mecânica comuns em próteses fixas convencionais não são mais necessários na odontologia contemporânea, filosofia que evita desgaste excessivo de estrutura dental sadia (EDELHOFF; SORENSEN, 2002; BÜCHI et al., 2014). O restabelecimento dos tecidos dentais perdidos por materiais restauradores, preservando-se ao máximo esmalte e 
dentina sadios, é um dos maiores desafios da odontologia minimamente invasiva na atualidade (MAGNE; BELSER, 2002; FRADEANI, 2004; BAZOS; NOVY; FULLER, 2008; MAGNE, 2011; MAGNE, 2015). Os autores comumente encontram pacientes com tratamentos desnecessariamente invasivos, com desgastes acentuados de tecidos dentais sadios para viabilizar tratamentos indiretos ou diretos mais amplos, como facetas. Apesar de a evolução da odontologia adesiva e dos sistemas cerâmicos ser de grande valia no arsenal odontológico, seu emprego em situações clínicas passivas de serem resolvidas com restaurações diretas limitadas à área de tecido dental perdido constituem em tratamento excessivamente invasivo.

Entendemos que a obtenção de resultados estéticos satisfatórios pode ser mais fácil ao se escolher tratamentos mais invasivos, mas é obrigação profissional capacitar-se para devolver função e estética ao paciente da maneira mais conservadora possível. Neste contexto, recomendamos fortemente a leitura do clássico artigo de Burke e Kelleher (2009) que aborda a necessidade de oferecermos a nossos pacientes aquilo que faríamos em nossa filha. Esta prática de preservar e cuidar de nossos pacientes como cuidaríamos de um parente próximo deve ser sempre levada em conta ao selecionarmos o tratamento a ser executado.

\section{REFERÊNCIAS}

ABU-BAKR, N. et al. Color stability of compomer after immersion in various media. Journal of Esthetic Dentistry, v. 12, n. 5, p. 258-263, 2000.

BAGHERI, R.; BURROW, M. F.; TYAS, M. Influence of food-simulating solutions and surface finish on susceptibility to staining of aesthetic restorative materials. Journal of Dentistry, v. 33, n. 5, p. 389-398, 2005.

BARATIERI, L. N. Dentística restauradora: fundamentos e possibilidades. São Paulo: Ed. Santos, 2001.

BAZOS, P.; MAGNE, P. Bio-emulation: biomimetically emulating nature utilizing a histo-anatomic approach; structural analysis. The European Journal of Esthetic Dentistry: Official Journal of The European Academy of Esthetic Dentistry, v. 6, n. 1, p. 8-19, 2011.

BÜCHI, D. et al. Minimally invasive rehabilitation of a patient with amelogenesis imperfecta. The International Journal of Esthetic Dentistry, v. 9, n. 2, p.13445, 2014.

BURKE, F. J.; KELLEHER, M. G. The "daughter test" in elective esthetic dentistry. Journal of Esthetic and Restorative Dentistry, v. 21, n. 3, p. 143146, 2009.

CHOI, M. S. et al. Changes in surface characteristics of dental resin composites after polishing. Journal of Materials Science Materials in Medicine, v. 16, $\mathrm{n}$. 4, p. 347-353, 2005. 
DAHL, J. E.; PALLESEN, U. Tooth bleaching--a critical review of the biological aspects. Critical Reviews in Oral Biology and Medicine, v. 14, n. 4, p. 292304, 2003.

DEMARCO, F. F. et al. Anterior composite restorations in clinical practice: findings from a survey with general dental practitioners. Journal of Applied Oral Science, v. 21, n. 6, p. 497-504, 2013.

DEMARCO, F. F. et al. Anterior composite restorations: A systematic review on long-term survival and reasons for failure. Dental Materials, v. 31, n. 10, p. 12141224, 2015.

EDELHOFF, D.; SORENSEN, J. A. Tooth structure removal associated with various preparation designs for posterior teeth. The International Journal of Periodontics \& Restorative Dentistry, v. 22, n. 3, p. 241-249, 2002.

ERTAS, E. et al. Color stability of resin composites after immersion in different drinks. Dental Materials Journal, v. 25, n. 2, p. 371-614, 2006.

FERRACANE, J. L. Resin composite - state of the art. Dental Materials, v. 27, n. 1, p. 29-38, 2011.

FONTES, S. T. et al. Color stability of a nanofil composite: effect of diferente immersion media. Journal of Applied Oral Science, v. 17, n. 5, p. 388-391, 2009.

FRADEANI. M. Esthetic Rehabilitation in Fixed Prosthodontics: Esthetic Analysis Volume 1. 1. ed. Quintessence Publishing, 2004. 352p.

HIRATA, R. Shortcuts em odontologia estética: uma nova visão sobre TIPS. 1. ed. São Paulo: Quintessence, 2016.

JOINER, A. The bleaching of teeth: a review of the literature. Journal of Dentistry, v. 34, n. 7, p. 412-419, 2006.

JOINER, A; LUO, W. Tooth colour and whiteness: a review. Journal of Dentistry, v. 67, p. 3-10, 2017.

KOSE, C. et al. Comparison of the Effects of In-office Bleaching Times on Whitening and Tooth Sensitivity: A Single Blind, Randomized Clinical Trial. Operative Dentistry, v. 41, n. 2, p. 138-145, 2016.

MAGNE, P.; BELSER, U. Bonded porcelain restorations in the anterior dentition: a biomimetic approach. 1.ed. Quintessence Pub Co., 2002. 406p. 
MAGNE, P. A. new approach to the learning of dental morphology, function, and esthetics: the "2D-3D-4D" concept. The International Journal of Esthetic Dentistry, v. 10, n. 1, p. 32-47, 2015.

MAIA, E. et al. The influence of two home-applied bleaching agents on enamel microhardness: an in situ study. Journal of Dentistry, v. 36, n. 1, p. 2-7, 2008.

MARAN, B. M. et al. Different light-activation systems associated with dental bleaching: a systematic review and a network meta-analysis. Clinical Oral Investigations, v. 23, n. 4, p. 1499-1512, 2019.

MATIS, B. A. et al. White diet: is it necessary during tooth whitening? Operative Dentistry, v. 40, n. 3, p. 235-240, 2015.

MCGUCKIN, R. S.; THURMOND, B. A.; OSOVITZ, S. Enamel shear bond strengths after vital bleaching. American Journal of Dentistry, v. 5, n. 4, p. 216222, 1992.

MJOR, I. A. et al. Placement andreplacement of restorations in general dental practice inlceland. Operative Dentistry, v. 27, n. 2, p. 117-123, 2002.

MORI, A. A. et al. Susceptibility to Coffee Staining during Enamel Remineralization Following the In-Office Bleaching Technique: An In Situ Assessment. Journal of Esthetic and Restorative Dentistry, v. 28, n. 1, p. 2331, 2016.

NOVÝ, B. B.; FULLER, C. E. The material science of minimally invasive esthetic restorations. Compendium of Continuing Education in Dentistry, v. 29, n. 6, p. 338-346, 2008.

PATEL, S. B. et al. The effect of surface finishing and storage solutions on the color stability of resin-based composites. The Journal of The American Dental Association, v. 135, n. 5, p. 587-594, 2004.

QUAGLIATTO, P. S. Clareamento dental e técnicas restauradoras para dentes clareados. São Paulo: Ed. Santos, 2020. 310p.

REZENDE, M. et al. Predictive factors on the efficacy and risk/intensity of tooth sensitivity of dental bleaching: A multi regression and logistic analysis. Journal of Dentistry, v. 45, p. 1-6, 2016.

REZENDE, M. et al. Clinical effects of exposure to coffee during at-home vital bleaching. Operative Dentistry, v. 38, n. 6, p. 229-236, 2013.

TITLEY, K. C. et al. Adhesion of a resin composite to bleached and unbleached human enamel. Journal of Endodontics, v. 19, n. 3, p. 112-115, 1993. 
UCHIDA, H. et al. Color stability of dental composites as a function of shade. The Journal of Prosthetic Dentistry, v. 79, n. 4, p. 372-377, 1998.

VAN MEERBEEK, B. et al. Relationship between bond-strength tests and clinical outcomes. Dental Materials, v. 26, n. 2, p.100-121, 2010. 